\title{
RAIL FAILURES FORECAST ON EAST SIBERIAN RAILWAY
}

\author{
Kovenkin Dmitriy \\ Federal State Budgetary Educational Institution "Irkutsk \\ State Transport University" \\ (FSBEI HE ISTU) \\ Irkutsk, the Russian Federation \\ kovenkin_pph@irgups.ru
}

\author{
Makhonina Ekaterina \\ Federal State Budgetary Educational Institution "Irkutsk \\ State Transport University" \\ (FSBEI HE ISTU) \\ Irkutsk, the Russian Federation \\ cat_181@mail.ru
}

\begin{abstract}
In this paper work, the effect of operational factors on the working sections of the ESRW track on the fatigue life of rails is investigated. Among the factors taken into account, the following are used: the effect of the axial load, the speed of rolling stock, the characteristics of the rolling stock, the parameters of the upper track structure, the parameters characterizing the rail track geometry. The algorithm used in the work allows one to take into account the effect on the contact fatigue strength of rails of the wear rate of the rail head, the effect of grinding rails, the thrust multiplicity, the driving regime and the complexity of operating conditions. The method of failure prediction used in this work makes it possible to calculate the tonnage, which can be passed through the given section before the repair of the track..
\end{abstract}

Keywords—rails, failure, forecast, tonnage, load, criterion.

\section{INTRODUCTION}

In connection with the constant growth of freight traffic of railways, axle loads of rolling stock and speeds of movement, the impact of rolling stock on the railway track also increase. As a result, the service life of the elements of the upper structure of the track is gradually decreasing $[1,2]$. Therefore, one of the priority tasks for track personnel is the task of increasing the duration of operation of railway track elements $[3,4]$, and, in particular, improving the rails service durability $[5,6,7]$. This problem cannot be successfully solved without creating effective methods for calculating the reliability and durability of the rails.

Improving the planning of track repairs, managing rail stocks for single and continuous shifts, planning for rail condition checks using non-destructive testing, assumes the use of methods for forecasting rails failures.

In this case the rail failure forecast makes it possible to solve a number of the following operational problems:

to study the impact of changes in operating conditions on the road section, including the effect of increasing the axle load or train speed on the fatigue life of rails;

to study the influence of various characteristics of the rolling stock and track on the fatigue life of rails;

to take into account the influence of the state of the rail track geometry on the fatigue life of rails;
- to analyze the existing inter-repair standards and identification of their compliance with changing operating conditions.

The output of rails for contact fatigue defects, along with the output of rails for wear, is one of the main criteria determining the lifetime of the track between overall repairs $[8,9]$.

\section{PROCEDURE FOR FORECASTING RAIL FAILURE}

The method of forecasting rail failure was applied in the work [10], which was investigated bythe head of the branch "Track and track facilities" Anatoly Yurievich Abdurashitov, the chief research officer of the branch "Track and track facilities" Alexandr Yakovlevich Koganand head of the laboratory "Continuous welded rail " JSC VNIIZhT Oleg Alexandrovich Suslov.

According to this document, it is possible to determine the following methodological order of forecasting the definition of tonnage, which can be passed through the calculated section before the exhaustion of the contact fatigue strength of rails:

to identify specific areas for monitoring the intensity of the exit of rails for defects of contact-fatigue origin;

to determine the main operational factors for the control sections (load and weight of the composition, axle load, speed and mode of motion, plan and profile of the track, type of track structure, actual state of the geometry of the track gauge);

to determine the value of the rail damage index in the experimental section $D_{0}$ and the average value of the axial load $P_{0}$ on the basis of mathematical modeling in conjunction with the results of operational observations;

based on the results of operational observations, the tonnage is determined at the control sections before the exhaust-contact fatigue strength of the rails $T_{0}=n_{0} \cdot P_{0}$ is depleted, where $\mathrm{n}_{0}$ is the number of axes passed through the inspection site prior to the removal of the rail;

as a result, for all categories of rails in the control sections, we obtain the fatigue resource passports, in which the 
values $D_{0}, P_{0}$ and the table of values of $T_{0}$ are indicated for different indicators of the rails output intensity (pcs / km);

in the future, if necessary, make a prediction of the service life of a particular section of the route, it is necessary to determine the main influencing operational factors for this section;

- $\quad$ on the basis of mathematical modeling in conjunction with the results of operational observations, the value of the rail damage index in the selected section $D_{1}$ and the average value of the axial load $\mathrm{P}_{1}$; are determined;

- $\quad$ by the relation $T_{1}=T_{0} \cdot \frac{D_{0}}{D_{l}} \cdot \frac{\mathrm{P}_{1}}{\mathrm{P}_{\mathbf{n}}} \cdot \frac{\tau_{0}}{\tau_{1}}$-define the value of $T_{l}$, which is the forecast tonnage, which determines the service life of the rails before their replacement. The values of $D_{0}$ and $P_{0}$ are taken according to the passport of the fatigue resource of the rail corresponding to that category or batch of rails, which was accepted as a calculation for the prediction, the values of $\tau_{0}$ and $\tau_{1}$ are taken depending on the climatic sections in the sections under study.

\section{EXECUTION OF THE FORECAST CALCULATION FOR THE SECTIONS OF THE ROUTE ON THE EAST-SIBERIAN RAILWAY}

For the forecast calculation, a track located within the boundaries of the East Siberian Railway was selected.

As a reference (reference) kilometer, a straight section of the up line is taken for $5190 \mathrm{~km}$. The design of the permanent way at this kilometer is a non-destructive track with a JBR-65 fastening, the longitudinal slope of the section is $3 \%$ (rise), the load strength is 98 million tons of gross / $\mathrm{km}$ per year. The climatic zone of the location of the estimated kilometer is defined both for the conditions of temperate climate, therefore $\tau=1$.
The length of straight lines in this section is all $1000 \mathrm{~m}$, therefore, the coefficient characterizing the share of the curves of the road sections is equal to $\varepsilon i=1000 / 1000=1$. The intensity of lateral wear $\mathrm{J}=0 \mathrm{~mm} /$ million tons gross for both rail threads. The track's content on the control kilometer according to the car's data is a good tracker. The grinding of the rails at this kilometer is carried out in full.

Characteristics of the rolling stock, the part of each type of rolling stock in the total flow is as follows:

- freight locomotives

- $\quad$ axle load $=25 \mathrm{t} /$ axis, share in the flow $=0.01$;

- $\quad$ areights

- $\quad$ axle load $=5,5$ t/axis, share in the flow $=0.05$;

- $\quad$ axle load $=23 \mathrm{t} / \mathrm{axis}$, share in the flow $=0.9$;

- $\quad$ passenger locomotives

- $\quad$ axle load $=22$ t/axis, share in the flow $=0.01$;

- $\quad$ passenger cars

- $\quad$ axleload $=16 \mathrm{t} / \mathrm{axis}$, share in the flow $=0.03$.

The train speed is $80 \mathrm{~km} / \mathrm{h}$ for both passenger and freight trains. Trains on the control kilometer follow the traction mode. The number of wheels with sliders passing through the control kilometer $\mathrm{km}$ was taken at $0 \%$. According to the fatigue passport for the rated category of rails we take $\mathrm{P}_{0}=23 \mathrm{t}$ $/$ axis, $\mathrm{D}_{0}=160 ; \mathrm{T}_{0}=850$ million tons gross (with the intensity of the removal of rails $4 \mathrm{pcs} / \mathrm{km}$ ). The results of the calculations are summarized in Table 1.

TABLE I. DETERMINATION OF THE FORECAST TONNAGE AT THE REFERENCE KILOMETER (STRAIGHT SECTION)

\begin{tabular}{|c|c|c|c|c|c|c|c|c|c|c|c|}
\hline \multirow{2}{*}{ 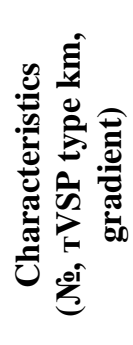 } & \multirow{2}{*}{ 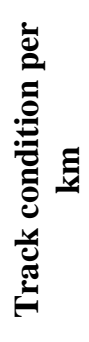 } & \multirow{2}{*}{ 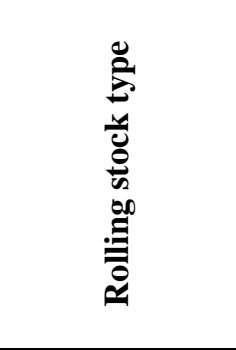 } & \multirow{2}{*}{ 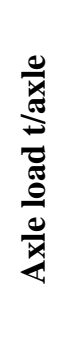 } & \multirow{2}{*}{ 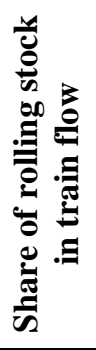 } & \multirow[b]{2}{*}{ 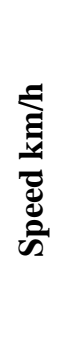 } & \multirow{2}{*}{ 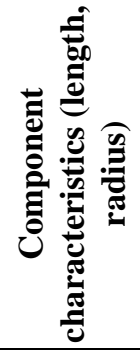 } & \multirow{2}{*}{ 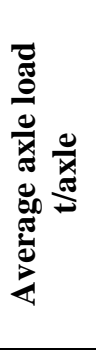 } & \multicolumn{2}{|c|}{ Value $D_{i}$} & \multicolumn{2}{|c|}{$\begin{array}{c}\begin{array}{c}\text { Forecasting } \\
\text { tonnage million } \\
\text { tons gross }\end{array} \\
\end{array}$} \\
\hline & & & & & & & & 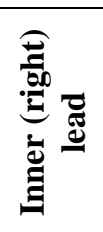 & 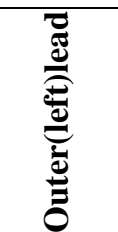 & 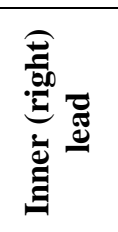 & 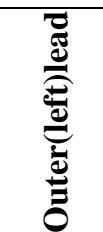 \\
\hline \multirow{5}{*}{ 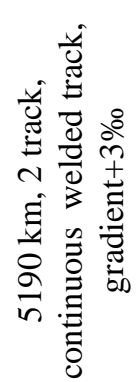 } & \multirow{5}{*}{ 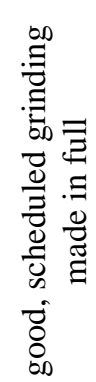 } & $\begin{array}{c}\text { freight } \\
\text { locomotives }\end{array}$ & 25 & 0.01 & \multirow{5}{*}{80} & \multirow{5}{*}{$\begin{array}{l}1000 \mathrm{~m} \text {, } \\
\text { straight }\end{array}$} & \multirow{5}{*}{21.93} & \multirow{5}{*}{180.55} & \multirow{5}{*}{180.90} & \multirow{5}{*}{718.06} & \multirow{5}{*}{716.67} \\
\hline & & freights (empty) & 5.5 & 0.05 & & & & & & & \\
\hline & & freights (loaded) & 23 & 0.9 & & & & & & & \\
\hline & & $\begin{array}{l}\text { passenger } \\
\text { locomotives }\end{array}$ & 22 & 0.01 & & & & & & & \\
\hline & & passenger cars & 16 & 0.03 & & & & & & & \\
\hline
\end{tabular}


As an estimated kilometer, an up-line section of $5186 \mathrm{~km}$ is accepted. The design of the permanent way at this kilometer is a continuous welded rail with a JBR-65 fastening, the longitudinal slope of the section is $3 \%$ (rise), the load strength is 98 million tons of gross / $\mathrm{km}$ per year. The length of straight lines is $37 \%$, the curves are $63 \%$, respectively.
According to the previous calculation, the following parameters $\mathrm{P} 0=21.93 \mathrm{t} /$ axis were adopted; $\mathrm{D} 0=180.55$ for the inner rail thread, D0 $=180.90$ for the outer rail thread; T0 correspondingly 718.06 and 716.67 million tons gross. All other basic data and calculation results are summarized in Table 2.

TABLE II. DETERMINATION OF THE FORECAST TONNAGE AT THE ESTIMATED KILOMETER (SECTION WITH CURVES)

\begin{tabular}{|c|c|c|c|c|c|c|c|c|c|c|c|c|c|c|}
\hline \multirow{2}{*}{ 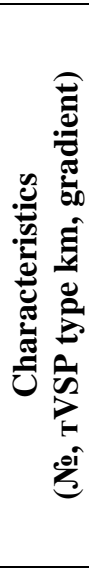 } & \multirow{2}{*}{ 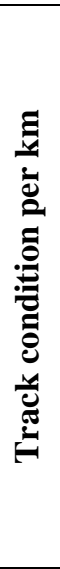 } & \multirow{2}{*}{ 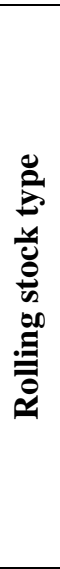 } & \multirow{2}{*}{ 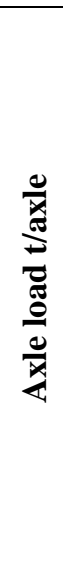 } & \multirow{2}{*}{ 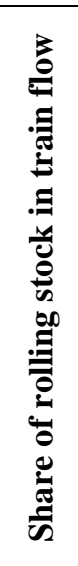 } & \multirow{2}{*}{ 苞 } & \multirow{2}{*}{ 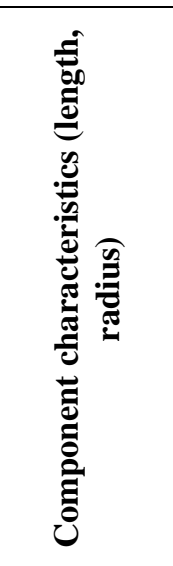 } & \multirow{2}{*}{ 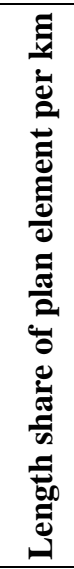 } & \multicolumn{2}{|c|}{$\begin{array}{c}\text { Intensity } \\
\text { side wear J, } \\
\text { mm/million } \\
\text { ton gross }\end{array}$} & \multirow{2}{*}{ 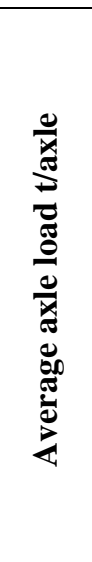 } & \multicolumn{2}{|c|}{ Value $D i$} & \multicolumn{2}{|c|}{$\begin{array}{l}\text { Forecasting } \\
\text { tonnage million } \\
\text { tons gross }\end{array}$} \\
\hline & & & & & & & & 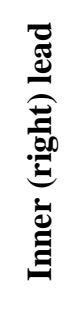 & 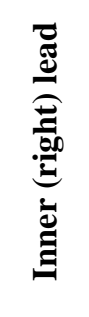 & & 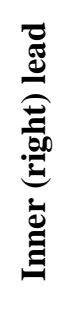 & 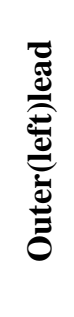 & 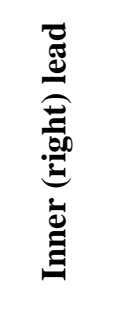 & 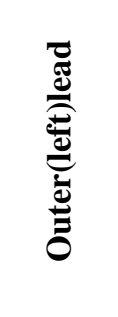 \\
\hline \multirow{12}{*}{ 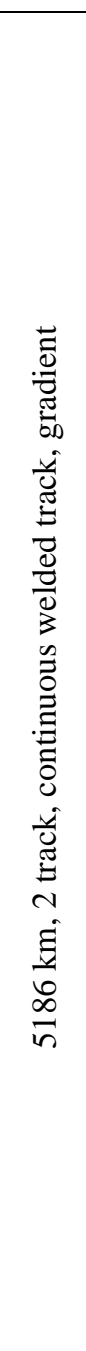 } & \multirow{12}{*}{ 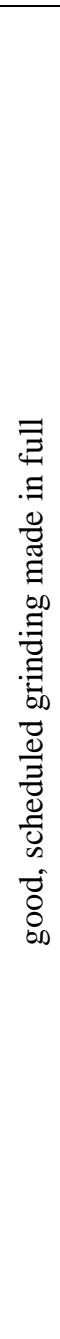 } & \multirow{6}{*}{ 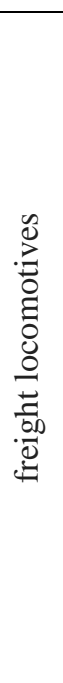 } & \multirow{6}{*}{25} & \multirow{6}{*}{0.01} & \multirow{6}{*}{80} & $\begin{array}{l}370 \mathrm{~m}, \\
\text { straight } \\
\text { line }\end{array}$ & 0.37 & 0.00 & 0.00 & \multirow{12}{*}{21.93} & \multirow{12}{*}{199} & \multirow{12}{*}{252} & \multirow{12}{*}{649.91} & \multirow{12}{*}{514.75} \\
\hline & & & & & & $\begin{array}{c}185 \mathrm{~m} \\
\mathrm{R}=835 \mathrm{~m}\end{array}$ & 0.19 & 0.00 & 0.02 & & & & & \\
\hline & & & & & & $\begin{array}{c}39 \mathrm{~m} \\
\mathrm{R}=301 \mathrm{~m}\end{array}$ & 0.04 & 0.00 & 0.03 & & & & & \\
\hline & & & & & & $\begin{array}{c}204 \mathrm{~m} \\
\mathrm{R}=595 \mathrm{~m}\end{array}$ & 0.20 & 0.00 & 0.03 & & & & & \\
\hline & & & & & & $\begin{array}{c}131 \mathrm{~m} \\
\mathrm{R}=1250 \mathrm{~m}\end{array}$ & 0.13 & 0.00 & 0.01 & & & & & \\
\hline & & & & & & $\begin{array}{c}71 \mathrm{~m} \\
\mathrm{R}=2000 \mathrm{~m}\end{array}$ & 0.07 & 0.00 & 0.01 & & & & & \\
\hline & & \multirow{6}{*}{ 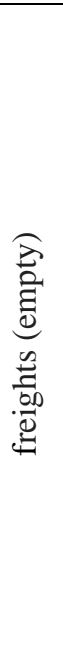 } & \multirow{6}{*}{5,5} & \multirow{6}{*}{0.05} & \multirow{6}{*}{80} & $\begin{array}{l}370 \mathrm{~m}, \\
\text { straight } \\
\text { line }\end{array}$ & 0.37 & 0.00 & 0.00 & & & & & \\
\hline & & & & & & $\begin{array}{c}185 \mathrm{~m} \\
\mathrm{R}=835 \mathrm{~m}\end{array}$ & 0.19 & 0.00 & 0.02 & & & & & \\
\hline & & & & & & $\begin{array}{c}39 \mathrm{~m} \\
\mathrm{R}=301 \mathrm{~m}\end{array}$ & 0.04 & 0.00 & 0.03 & & & & & \\
\hline & & & & & & $\begin{array}{c}204 \mathrm{~m} \\
\mathrm{R}=595 \mathrm{~m}\end{array}$ & 0.20 & 0.00 & 0.03 & & & & & \\
\hline & & & & & & $\begin{array}{c}131 \mathrm{~m}, \\
\mathrm{R}=1250 \mathrm{~m}\end{array}$ & 0.13 & 0.00 & 0.01 & & & & & \\
\hline & & & & & & $\begin{array}{c}71 \mathrm{~m} \\
\mathrm{R}=2000 \mathrm{~m}\end{array}$ & 0.07 & 0.00 & 0.01 & & & & & \\
\hline
\end{tabular}




\begin{tabular}{|c|c|c|c|c|c|c|c|c|c|c|c|c|c|c|}
\hline \multirow{2}{*}{ 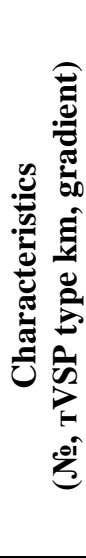 } & \multirow{2}{*}{ 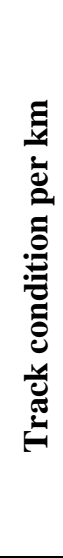 } & \multirow{2}{*}{ 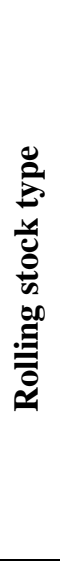 } & \multirow{2}{*}{ 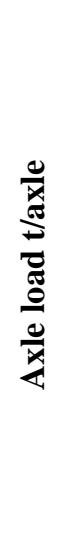 } & \multirow{2}{*}{ 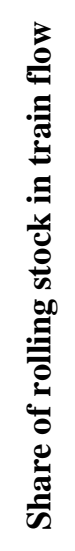 } & \multirow{2}{*}{ 㿣 } & \multirow{2}{*}{ 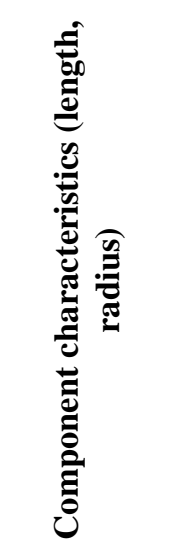 } & \multirow{2}{*}{ 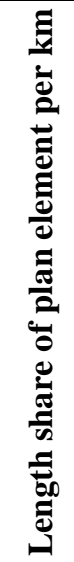 } & \multicolumn{2}{|c|}{$\begin{array}{c}\text { Intensity } \\
\text { side wear J, } \\
\text { mm/million } \\
\text { ton gross }\end{array}$} & \multirow{2}{*}{ 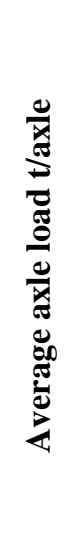 } & \multicolumn{2}{|c|}{ Value $D i$} & \multicolumn{2}{|c|}{$\begin{array}{l}\text { Forecasting } \\
\text { tonnage million } \\
\text { tons gross }\end{array}$} \\
\hline & & & & & & & & 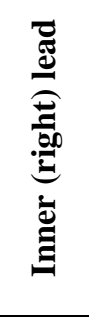 & 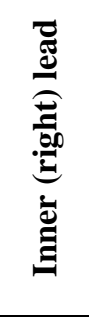 & & 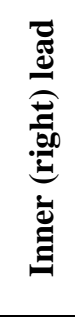 & 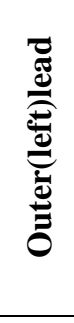 & 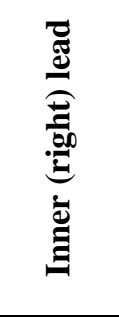 & 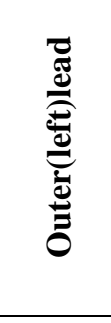 \\
\hline & & \multirow{6}{*}{ 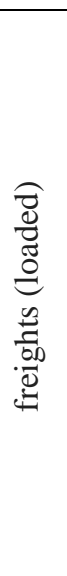 } & \multirow{6}{*}{23} & \multirow{6}{*}{0.9} & \multirow{6}{*}{80} & $\begin{array}{l}370 \mathrm{~m}, \\
\text { straight } \\
\text { line }\end{array}$ & 0.37 & 0.00 & 0.00 & & & & & \\
\hline & & & & & & $\begin{array}{c}185 \mathrm{~m} \\
\mathrm{R}=835 \mathrm{~m}\end{array}$ & 0.19 & 0.00 & 0.02 & & & & & \\
\hline & & & & & & $\begin{array}{c}39 \mathrm{~m} \\
\mathrm{R}=301 \mathrm{~m}\end{array}$ & 0.04 & 0.00 & 0.03 & & & & & \\
\hline & & & & & & $\begin{array}{c}204 \mathrm{~m}, \\
\mathrm{R}=595 \mathrm{~m}\end{array}$ & 0.20 & 0.00 & 0.03 & & & & & \\
\hline & & & & & & $\begin{array}{c}131 \mathrm{~m}, \\
\mathrm{R}=1250 \mathrm{~m}\end{array}$ & 0.13 & 0.00 & 0.01 & & & & & \\
\hline & & & & & & $\begin{array}{c}71 \mathrm{~m}, \\
\mathrm{R}=2000 \mathrm{~m}\end{array}$ & 0.07 & 0.00 & 0.01 & & & & & \\
\hline \multirow{12}{*}{ 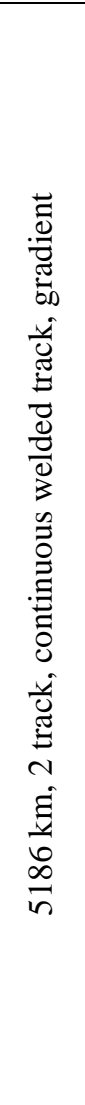 } & \multirow{12}{*}{ 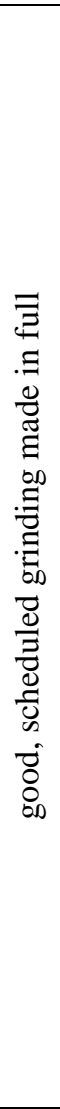 } & \multirow{6}{*}{ 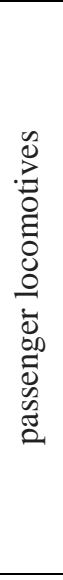 } & \multirow{6}{*}{22} & \multirow{6}{*}{0.01} & \multirow{6}{*}{80} & $\begin{array}{c}370 \mathrm{~m}, \\
\text { straight } \\
\text { line }\end{array}$ & 0.37 & 0.00 & 0.00 & \multirow{12}{*}{21.93} & \multirow{12}{*}{199} & \multirow{12}{*}{252} & \multirow{12}{*}{649.91} & \multirow{12}{*}{514.75} \\
\hline & & & & & & $\begin{array}{c}185 \mathrm{~m} \\
\mathrm{R}=835 \mathrm{~m}\end{array}$ & 0.19 & 0.00 & 0.02 & & & & & \\
\hline & & & & & & $\begin{array}{c}39 \mathrm{~m} \\
\mathrm{R}=301 \mathrm{~m}\end{array}$ & 0.04 & 0.00 & 0.03 & & & & & \\
\hline & & & & & & $\begin{array}{c}204 \mathrm{~m}, \\
\mathrm{R}=595 \mathrm{~m}\end{array}$ & 0.20 & 0.00 & 0.03 & & & & & \\
\hline & & & & & & $\begin{array}{c}131 \mathrm{~m}, \\
\mathrm{R}=1250 \mathrm{~m}\end{array}$ & 0.13 & 0.00 & 0.01 & & & & & \\
\hline & & & & & & $\begin{array}{c}71 \mathrm{~m}, \\
\mathrm{R}=2000 \mathrm{~m}\end{array}$ & 0.07 & 0.00 & 0.01 & & & & & \\
\hline & & \multirow{6}{*}{ 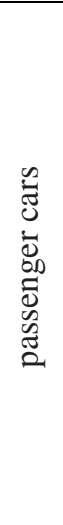 } & \multirow{6}{*}{16} & \multirow{6}{*}{0.03} & \multirow{6}{*}{80} & $\begin{array}{c}370 \mathrm{~m}, \\
\text { straight } \\
\text { line }\end{array}$ & 0.37 & 0.00 & 0.00 & & & & & \\
\hline & & & & & & $\begin{array}{c}185 \mathrm{~m} \\
\mathrm{R}=835 \mathrm{~m}\end{array}$ & 0.19 & 0.00 & 0.02 & & & & & \\
\hline & & & & & & $\begin{array}{c}39 \mathrm{~m} \\
\mathrm{R}=301 \mathrm{~m}\end{array}$ & 0.04 & 0.00 & 0.03 & & & & & \\
\hline & & & & & & $\begin{array}{c}204 \mathrm{~m} \\
\mathrm{R}=595 \mathrm{~m}\end{array}$ & 0.20 & 0.00 & 0.03 & & & & & \\
\hline & & & & & & $\begin{array}{c}131 \mathrm{~m}, \\
\mathrm{R}=1250 \mathrm{~m}\end{array}$ & 0.13 & 0.00 & 0.01 & & & & & \\
\hline & & & & & & $\begin{array}{c}71 \mathrm{~m}, \\
\mathrm{R}=2000 \mathrm{~m}\end{array}$ & 0.07 & 0.00 & 0.01 & & & & & \\
\hline
\end{tabular}




\section{CONCLUSIONS FROM THE RESULTS OF CALCULATIONS}

It can be seen from the data in Table 1 (straight section), the forecast tonnage, which can be passed along the rails at the control kilometer, will be about 720 million tons of gross on both rail threads. Accordingly, the estimated service life of rails on this site in years will be equal to $720 / 98=7.3$ years.

According to the data in Table 2 (section with curves), the forecast tonnage, which can be passed along the rails at the calculated kilometer, will be about 650 million tons of gross yarn per inner thread, about 515 million tons of outer thread. The forecasted service life in years will be $650 / 98=6.6$ years and $515 / 98=5.3$ years, respectively.

Comparing the two sections, we can conclude that the predicted service life in years before the onset of the intensity of the exit of rails on the straight section is greater; therefore, the yield of the rails will come later than on the curved path section. Data on the forecast tonnage must be taken into account for the purpose of overhauling the track.

\section{References}

[1] D. Kovenkin, R. Kupko, "Intermediate bonds on the mountain-pass section," Path and track facilities, vol. 6, pp. 2-7, June 2014.

[2] D. Kovenkin, V.Podverbniy, R. Kupko and O. Khauk, "The roadway machines using maintenance technology of the mountain-pass district of slyudyanka track division," Modern technologies. System analysis. Modeling, vol. 1(41), pp. 186-192, 2014.

[3] D. Kovenkin, V.Podverbniy, "Diagram of the formation stages of the life cycle of the top structure of a railway track," Transport infrastructure of the Siberian region, vol. 6, pp. 548-552, October 2015.

[4] V. Beltyukov, I. Simonyuk and A. Andreev, "Cost forecasting and optimization - the basis of repair planning," Path and track facilities, vol. 2, pp. 16-20, February 2014.

[5] A. Abdurashitov, "About increase of operational durability of rails," Railway transport: scientific-theoretical and technical-economic journal, vol. 9, pp. 57-60, 2012.

[6] S. Chernyak, V. Dementyev and L. Korneva, "Rails of increased operation durability in Siberia," Modern technologies. System analysis. Modeling, vol. 2(18), pp. 186-192, 2008.

[7] A. Ilyinih, V. Grigoriev "Modes of resistance and limits fatigue," World of Transport and Transportation, vol. 1, pp. 58-62, June 2007.

[8] A. Abdurashitov, "Single track removal as major criteria of railway track reconstruction assignment," Bulletin of the Institute of natural monopolies: railway engineering, vol. 2(30), pp. 26-31, 2015.

[9] "Technical conditions for the reconstruction (modernization) and repair of the railway track," JSC "Russian Railways", approved 18.01.2013 г. № 75r, pp. 41-45, 2013.

[10] A. Abdurashitov, A. Kogan and O. Suslov "Method for predicting rail failure," All-Russian Scientific Research Institute of Railway Transport, pp. 1-20, 2013. 